\title{
A Telecooperation Management System for Secure Data Conferencing in Distributed Product Development Processes
}

\author{
Prof. Dr.-Ing. F.-L. Krause, \\ Dipl.-Ing. H. Jansen, \\ Dipl.-Inform. R. Schultz, \\ Cand.-Inform. H. Gärtner \\ Fraunhofer Institute for Production Systems and Design Technology - IPK Berlin \\ e-mail: [first name]". "[last name]"@ipk.fhg.de"
}

\begin{abstract}
Telecooperation is a technology which fulfills the communication requirements arising from global distributed product development. Implementations of the T.120 standard take the heterogeneous environment into account but do not sophistically solve security and ergonomic problems. The Telecooperation Management System (TCMS), developed by the Fraunhofer Institute for Production Systems and Design Technology, enhances T.120 implementations for their successful use in distributed product development.
\end{abstract}

Key words: distributed product development, process chain, computer aid, telecooperation, teamwork, application sharing, data security, Telecooperation Management System (TCMS)

\section{SITUATION OF THE DISTRIBUTED PRODUCT DEVELOPMENT}

As a result of global competition, international consolidation and cooperation of companies is leading to the distributed development of products. Comprehensive use of computer aided technologies in all phases of the product development process and worldwide availability of networks allow the sharing of digital information between different company sites $/ 1 /$.

The original version of this chapter was revised: The copyright line was incorrect. This has been corrected. The Erratum to this chapter is available at DOI: 10.1007/978-0-387-35492-7_50 
These assumptions allow the application of information and communication technologies (ICT), particularly computer supported cooperative work (CSCW) and telecooperation systems. Examples of these systems are electronic mail, chat, white boarding, application sharing, and video conferencing.

Because of the optimised exchange of information between different team members in the development process, the organisational benefits of telecooperation are $/ 2 /$ :

- reducing the number of changes,

- reducing development costs and time,

- increasing the quality.

In particular, the use of application sharing systems will bring benefits to the product development process, because these systems allow the common, distributed use of any computer program. If a CAD program is shared in a data conference, all members can see the same view of the model displayed, they can mark areas and modify the model. Therefore, a flexible and comfortable cooperation between team members is independent of their location in the world.

But, high requirements from application sharing systems on the network infrastructure lead to several problems. Reasons for the problems are:

- heterogeneous hard- and software in different networks,

- interoperability of the telecooperation systems used,

- different security requirements and infrastructure as well as

- lack of user friendliness of the systems.

The first two points are the result of company specific guidelines during the construction of their network infrastructure. Only the consideration of standards allows the communication between systems of different hardware manufacturers. A promising way to handle these problems is the use of TCP/IP based systems programmed in Java. This is due to the fact that TCP/IP and Java are designed to work in a heterogeneous environment. For application sharing systems, the most common standards are known as H.323, H.320 and T.120/3/. The H.32x standards include the T.120 standard and are designed for video and audio conference via ISDN (H.320) or IP based networks (H.323)/4/. The T.120 standard defines the communication for application sharing, white boarding, remote pointing, chat and file transfer $/ 5 /$. 
Platform-independent use of T.120 systems is possible because Microsoft (NetMeeting), SUN Microsystems (SunForum), Silicon Graphics (SGIMeeting) and IBM (DC-Share) offer implementation software and most of them are available for free. With several million downloads, these systems are wide spread, but are not used for inter-company communication.

The third point in the list of problems concerns the security policy of the companies. During product development, exchanged data is very important for the companies. For example, the models represent development knowhow or the style of the planned product. Therefore every company defines a security policy where, among other things, the security requirements for telecooperation are specified.

Telecooperation is only possible between companies, if the required resources are permitted in all companies involved. Figure 1 shows the generally available resources for telecooperation and the three sets (A, B and C) of company specific resources. Telecooperation between companies A and $B$ is only possible with the resources in the intersection A $\cap$ B. Therefore, telecooperation systems must allow company specific configuration on one hand, but enable flexible communication with other systems on the other hand.

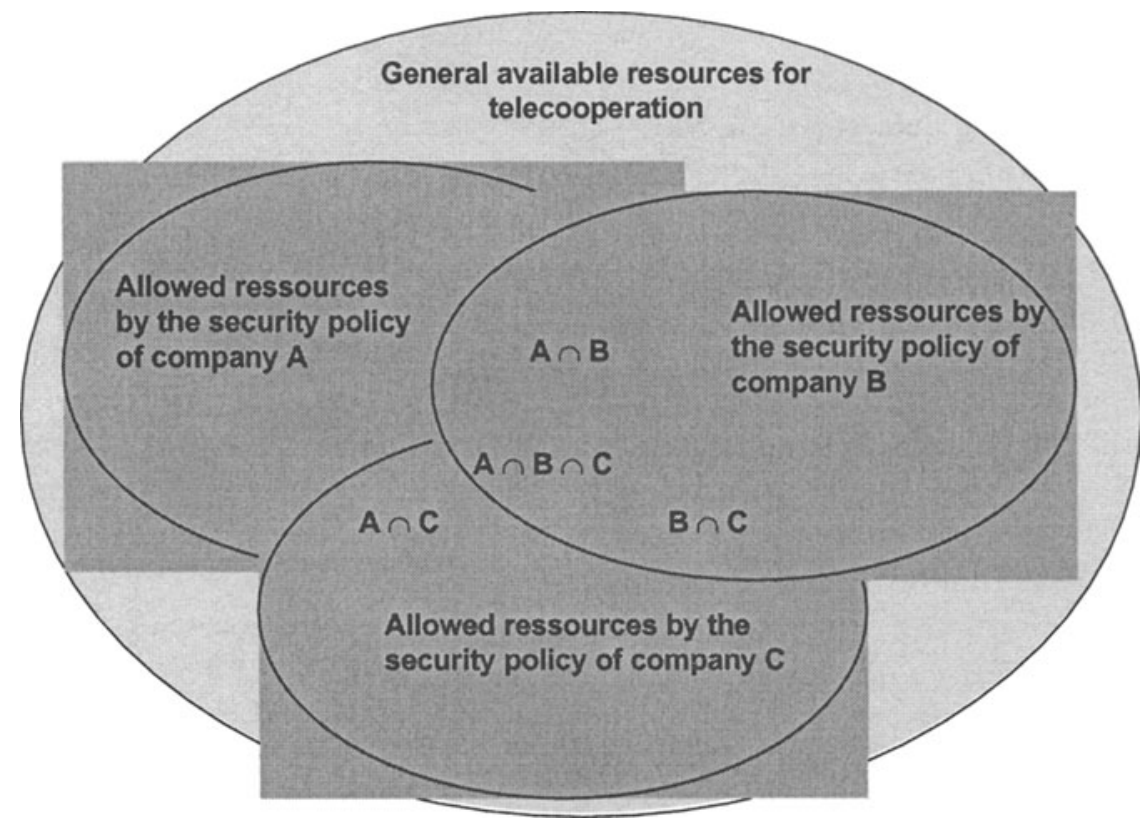

Figure 1: Set of allowed resources for telecooperation 
The point to point connection between the conference computers due to IP based teleconference systems leads to problems concerning the security infrastructure. These issues include:

- the definition of permissions for every possible connection results in many open ports for many IP-addresses in the firewall,

- the use of unofficial or dynamic IP-addresses is not possible,

- the administration of the firewall requires a lot of time and

- many and not well ordered entries are the reason for loosing the overview.

From an ergonomic viewpoint, the use of telecooperation systems is complex for an engineer. If the user wants to establish a conference, he has to know the IP address of the computer of his conference partner. He must obtain the IP address and is, therefore required to call his contact for this information. Figure 2 shows the IP based invitation of a conference partner with the T.120 system MS NetMeeting.

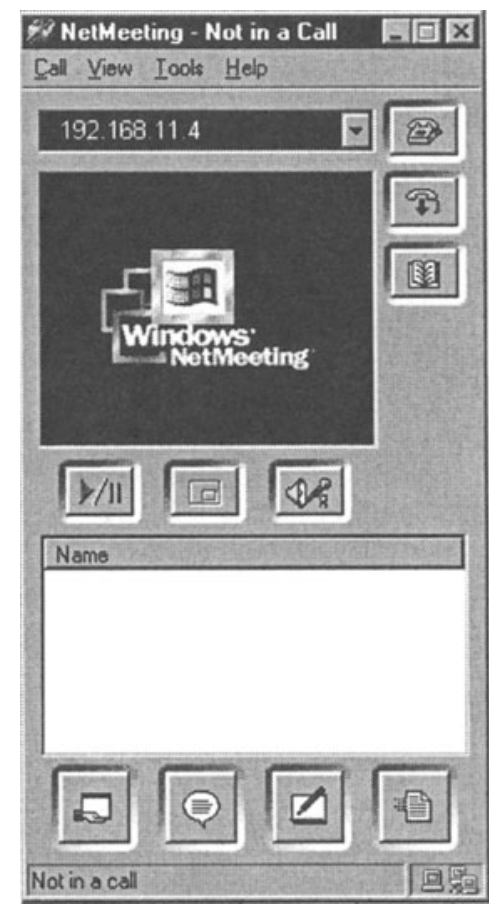

Figure 2: Invitation to conference with NetMeeting

Nowadays, the third and the fourth point are not sophistically solved by the T.120 implementations. The reasons for this are, on the one hand the required end-to-end-IP-connection between conference computers which results in the creation of great holes in the firewalls. On the other hand, the 
IP-based invitation of conference partner can not be supported by address books, because the information is always changing if the chosen person works in a pool or the dynamic host configuration protocol (DHCP) is used.

This paper describes the concept of the telecooperation management system (TCMS) which provides a solution for several teleconference protocols. The exemplary supported standard is T.120 for two reasons: in the product development process, pure application sharing is the better solution compared to application sharing with video conferencing. Video reduces the space on the screen, where the $\mathrm{CAD}$ window displays the model. Moreover, video and audio occupy the bandwidth on networks and have a negative effect on the performance of the shared program.

\section{ARCHITECTURE OF THE TELECOOPERATION MANAGEMENT SYSTEM}

To fulfill the requirements of distributed product development, the solution must be platform independent, scalable, and must support the company specific security policy. Therefore the concept of the TCMS requires a TCMS on both sides and is implemented in JAVA. Figure 3 shows how the TCMS splits the data stream in an internal and an external part.

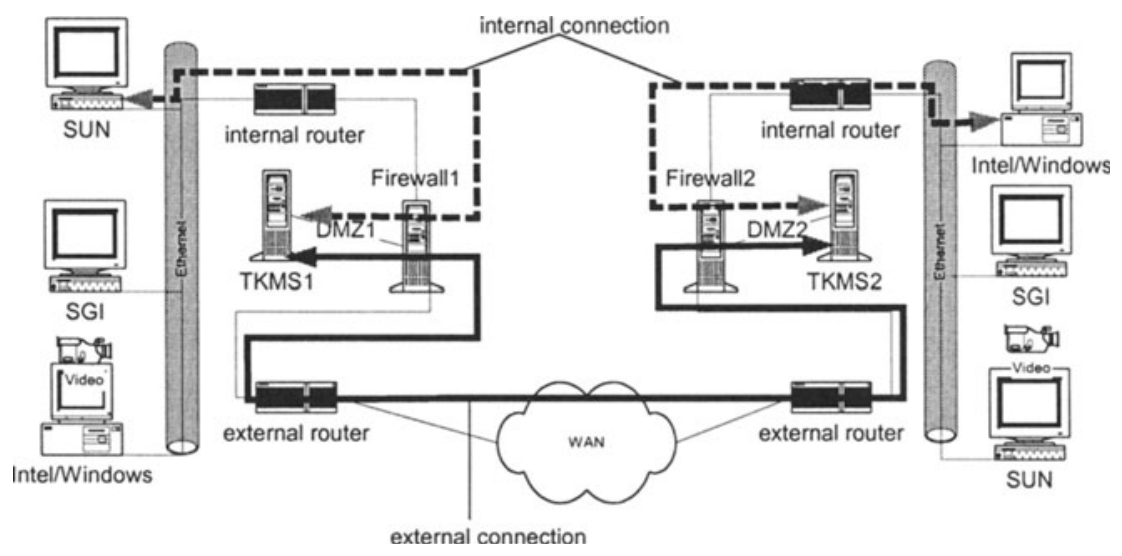

Figure 3: Telecooperation with the TCMS

The communication between every internal user workstation requires only connections to the demilitarised zone in the firewall. The external part of a teleconference requires only one connection to the external TCMS trough the firewall for every possible conference. 
In order to keep the company security and network configuration secret, both TCMS negotiate the available resources before a conference and decide by conference parameter if it is allowed. The different modules of the scalable TMCS are shown in Figure 4.

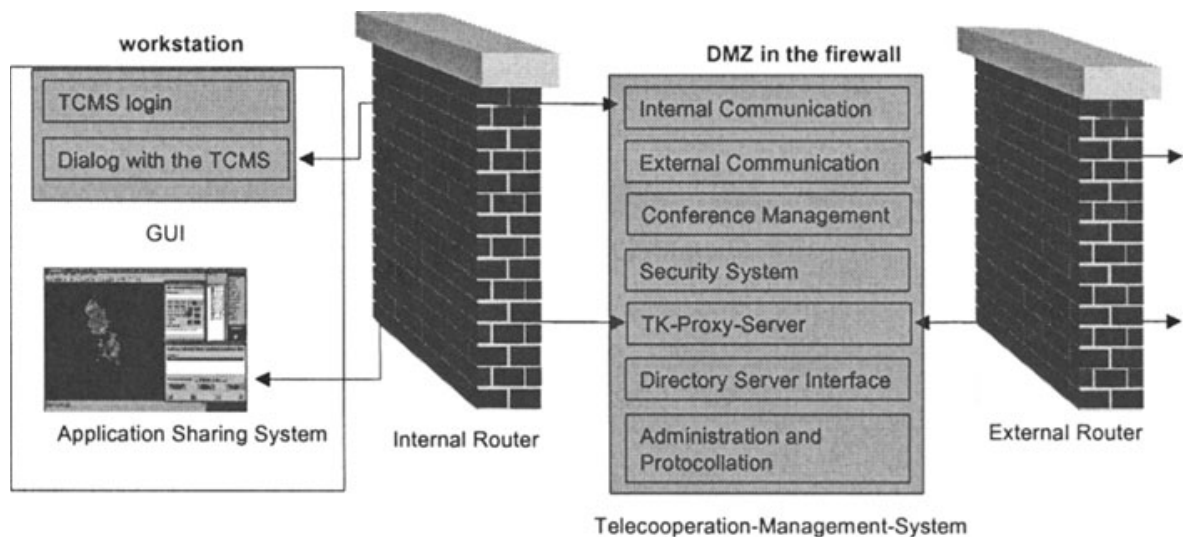

Figure 4: Architecture of the TCMS

The left side of the picture represents a user workstation with the TCMS user interface and the right side the demilitarised zone in the firewall with the modules of the TCMS.

The graphical user interface $(G U I)$ is implemented using web technologies. The dialogs for login and for establishing a conference are either HTML pages or JAVA applets. The advantages are, on the one hand, the low installation expense because, apart from a web browser on the user computer, no additionally software is necessary. The user interface and the TCMS have to be installed only one time for the entire company. On the other hand, is the ability of using a common browser wide spread, whereby the user qualification is reduced to the functionality of the system.

The Internal Communication realises the communication between the TCMS and the user interface. Login and conference requests from the internal network are received and conference invitation from external partners are delivered.

The External Communication realises the communication with other external TCMS. Because of security aspects, only conferences between TCMS are possible. During the communication, both TCMS exchange the conference parameter and negotiate the coding algorithms and ports for the communication. 
The main module of the TCMS is the Conference Management. This module controls the required resources for incoming and outgoing conferences and decides by conference parameter if a conference will take place or not. These parameters are:

- internal user and internal conference computer,

- external user

- required conference system and

- date and time of the conference.

The Security System realises the verification of conference parameters and the coding of all transferred data between internal and external TCMS and user workstation and TCMS.

For every required teleconference protocol, a Proxy Server can be implemented and integrated in the TCMS. The advantages of this solution are the independence of teleconference protocols used, the hiding of the internal network infrastructures and the reduction of holes in the firewall to one port for the IP address of the proxy server. The implemented proxy server supports the T.120 protocol on the platforms MS Windows, Sun Solaris, SGI IRIX and IBM AIX.

The Directory Server Interface allows the use of information stored in a companies own directory services.

The Administration and Record allows the definition of conference permissions and the comprehensive recording of all conferences, attacks, and errors.

\section{ESTABLISHING A TELECONFERENCE WITH THE TCMS}

The TCMS makes telecooperation between two companies or sites in the distributed product development possible because it allows the realisation of company own security policy by enhancing the T.120 standard by several security features. The proxy server reduces the open ports in the firewall, allows network address translation and encrypts the transferred data using the secure socket layer (SSL) technology. 
The Conference Management allows the invitation of a conference partner by using his e-mail address, shown in figure 5 . The conference request is transferred to the internal TCMS where permission is verified. If permission is given, the internal TCMS starts a connection with the external TCMS and both negotiate the resources for the conference. Finally, the external TCMS starts a connection to the invited user, because links between user and the IP address of the computer are defined during the user login.



Figure 5: Inviting a conference partner

If the invited conference partner accepts the invitation and permission for the conference is given, a window is shown to the initiator of the process so that he should start the chosen teleconference system and connect to the internal proxy server of the TCMS, shown in figure 6. If a domain name service is available in the network, the IP address of the proxy server can be replaced by the name of the computer where the TCMS is running, for example TCMS.supplier.com. 




Figure 6: Invitation accepted

The internal proxy server is waiting for the connection from the user computer and forwards all incoming data to the external proxy server which forwards the data to the workstation of the conference partner. A teleconference is now established. If any error occurs before or during a conference, the TCMS will provide the administrator and the user with context specific information to solve the problem.

\section{SUMMARY}

Distributed product development in global markets requires efficient and secure communication between partners. This demands a small amount of time and money for the installation, adaptation and maintenance of the necessary infrastructure. Not only small and medium sized enterprises need to be able to communicate with several partners via low budget networks like the internet, but the transport of secret information on public networks leads to additional costs for the integration of high security technologies to save the data.

Teleconferencing with TCMS solves all the mentioned problems, because available features are:

- transparent establishment of a conference (only e-mail is required),

- reliable user and server authentication before a conference,

- hidden infrastructure with a minimum opened firewall

- secure data transmission (secured by SSL or IPsec),

- conference parameter based release of resources,

- realisation of company specific security policy,

- easy to install (zero maintenance solution),

- easy to configure and administrate and

- easy to use. 
The Telecooperation Management System provides a significant reduction of requirements for flexible and secure teleconferences. Platform independence and comprehensive configuration possibilities allow the integration of the TCMS in any existing infrastructure to fulfil the company specific security policy. Up to date authentication and coding technologies allow conferencing over public networks. The modular architecture and the proxy concept allow the easy adaptation of the TCMS forced by changed telecooperation protocols.

\section{REFERENCES}

/1/ Spur, G.; Krause, F.-L.: Das virtuelle Produkt - Management der CADTechnik. Carl Hanser Verlag, München, Wien 1997

/2/ Krause, F.-L.; Schultz, R.: Integration praxisorientierter Telekooperationssysteme in verteilte Produktentwicklungsprozesse eines Automobilhersteller/-zulieferer-Netzwerks. In: VDI-Berichte 1537: Der Ingenieur im Internet - Erfolgreiche Anwendungen in der Industrie. Tagung Karlsruhe, 27./28. März 2000. VDI Verlag, Düsseldorf 2000, S. $145-168$

13/ N.N: International Telecommunication Union. URL: http://www.itu.int/home/index.html, 2000

14/ N.N A Primer on the H.323 Series Standard. URL: http://www.databeam.com/h323/h323primer.html, 2000

15/ N.N A Primer on the T.120 Series Standard. URL: http://www.lotus.com/products/sametime.nsf/standards/8DDB25B6C08E70 E5852566640072FCD2, 2000

\section{BIOGRAPHY}

Prof. Dr.-Ing. Frank-Lothar Krause, born in 1942, studied Production Technology at the Technical University of Berlin. In 1976, he became Senior Engineer for the CAD Group at the Institute for Machine Tools and Production Technology (IWF) of the TU Berlin and earned his doctorate under Prof. Spur. Since 1977, he has been Director of the Virtual Product Development Devision at the Fraunhofer Institute for Production Systems and Design Technology (IPK Berlin). He earned the qualification as a university lecturer in 1979 and has been University Professor for Industrial Information Technology at the IWF of the TU Berlin since 1990.

Dipl.-Ing. Helmut Jansen, born in 1946, studied Electrotechnical Science at the Technical University of Berlin. In 1977 he joined the department of Industrial Information Technology at the Institute of Machine 
Tools and Manufacturing Technology of the Technical University of Berlin. In 1980 he joined the Virtual Product Development Division of the Fraunhofer Institute for Production Systems and Design Technology (IPK Berlin). Since 1985 he is head of the department Information Technology and has worked in several national and international research projects in the field of Man-Machine-Communication, Pattern Recognition and Virtual Product Creation.

Dipl.-Inform. Ralph Schultz, born in 1965, studied Computer Science at the Technical University of Berlin. In 1995 he joined the department of Industrial Information Technology at the Institute of Machine Tools and Manufacturing Technology of the Technical University of Berlin. Since 1999 he is joining the Information Management Department at the Virtual Product Development Division of the Fraunhofer Institute for Production Systems and Design Technology (IPK Berlin). He has worked in several national and international research projects in the field of telecommunications and security in distributed product development.

Cand.-Inform. Hendrik Gärtner, born in 1971, studied Computer Science at the Freie Universität of Berlin. Since 1999 he is joining the Information Management Department at the Virtual Product Development Division of the Fraunhofer Institute for Production Systems and Design Technology (IPK Berlin). He has worked in national and international research projects in the field of telecommunications and security. 